\title{
The role of attack and defense semantics in skilled players' memory for chess positions
}

\author{
STUART J. MCGREGOR and ANDREW HOWES \\ Cardiff University, Cardiff, Wales
}

\begin{abstract}
There is much evidence that chess skill is based on chunks in memory that represent parts of positions from previously encountered games. However, the content of these chunks is a matter for debate. According to one view, (1) the closer two pieces are to each other on a board (proximity), the more likely they are to be in the same chunk, and (2) skilled players encode the precise locations of pieces. An alternative view is that what information is encoded in a chess chunk is determined more by processing of the attack/defense relations during evaluation. In three experiments, participants evaluated positions and completed recognition tests. Experiment 1 supported the view that expert players make more use of attack/defense relations than of locations of pieces in a recognition test. Experiments 2 and 3 demonstrated that, for both long and short presentation times, expert players' recognition for a piece within a position was primed more by a piece related by attack or defense than by a piece merely proximal. These findings challenge theories of expertise for chess that assume a primary role for proximity and location in determining which pieces are grouped together in memory.
\end{abstract}

It has long been established that the difference between expert and novice performance in chess is due to an expert's ability to rapidly recognize situations and retrieve appropriate actions. According to Chase and Simon (1973a, 1973b), a chess expert's ability to recognize situations is based on the presence in memory of many thousands of chunks of information, each of which encodes a specific arrangement of pieces representing part of a board position. They proposed that throughout a lengthy period of playing experience, skilled players had encoded a large number of such chunks in long-term memory (LTM). Chase and Simon's theory was an application of Miller's (1956) chunking theory of memory, which stated that multiple items could be stored in permanent memory as one item or chunk.

Chunking theory has been supported by a series of recall experiments (Chase \& Simon, 1973a, 1973b; de Groot, 1965; Gobet \& Simon, 1996a, 1996b, 1996d; Goldin, 1978). De Groot (1965) was the first to report that more highly skilled players recalled briefly presented $(2-15 \mathrm{sec})$ chess positions significantly better than did less skilled players. Indeed, the more highly skilled (master-level) players used in de Groot's (1965) study recalled around $50 \%$ more pieces from game positions than did the less skilled (expert) players. This effect of skill level on recall memory has been replicated by numerous studies (e.g., Chase \& Simon, 1973a, 1973b; Gobet \& Simon, 1998; Goldin, 1978).

According to Chase and Simon (1973a, 1973b), the recall effect of skill occurs because more highly skilled

Correspondence concerning this article should be addressed to A. Howes, School of Psychology, Cardiff University, P.O. Box 901, Cardiff CF10 3YG, Wales (e-mail: howesa@ cardiff.ac.uk). players hold greater numbers of chess chunks in LTM and are more likely to be able to match an LTM chunk to a group of pieces from any game position. Consequently, an expert is more likely to be able to retrieve a number of chunks from short-term memory, whereas a novice player without the matching chunks can recall only a number of single pieces. Evidence supporting this view was provided by recall studies using random chess positions. When positions generated randomly (as opposed to positions borrowed from game play) were used in a recall experiment, Chase and Simon (1973a, 1973b) found no effect of skill level on performance. This, they argued, was due to the fact that the more highly skilled players were not able to match chunks of pieces in LTM to any of the arrangements of pieces in the random positions. This finding has more recently been qualified by Gobet and Simon (1996b), who revealed a small effect of skill for recall of random positions, when the results of several studies were collated in a meta-analysis. They proposed that this small effect could be explained by the occasional presence of gamelike configurations within random positions.

Chase and Simon's (1973a, 1973b) chunking theory has been extended by Gobet and Simon (1996d), who proposed their template theory of expert chess memory. They argued that high-level expert players possess memory structures (templates) that represent whole board positions of pieces, as opposed to merely parts of positions. Furthermore, templates contain empty slots (squares) at which any information (chess pieces) can be instantiated. All other pieces in the memory template are described as core pieces that delineate each template. Gobet and Simon (1996d) claimed that the more powerful template structures are required to account for the full extent of high-level players' memory performance. An excellent 
feature of Gobet and Simon's (1996d) template theory is that it is grounded in a fully specified computational model that makes precise, unambiguous predictions.

Chunking theory and template theory have been implemented in the computational models MAPP (Simon \& Gilmartin, 1973) and CHREST (de Groot \& Gobet, 1996; Gobet \& Simon, 1996d), which modeled the recall performance of expert chess players. CHUMP (Gobet \& Jansen, 1994) modeled move selection on the basis of the association between chunks and templates with stored move choices and was reported to be capable of weak chess play.

Chunking theory and template theory are well specified, precise theories that have been shown to be consistent with the results of many studies. However, two questions remain: (1) Which chess pieces are combined into chunks with which others, and (2) are chunks encoded with information specific to a particular board location (the square on the board)? Answers to these questions are important for two reasons. First, they can inform theories of the mechanisms by which chunks are created, as well as the relation between what is remembered and the problem-solving processes used to evaluate positions. Second, it is generally assumed that the time it takes to acquire expertise is related to the number of chunks required for expertise (Simon \& Gilmartin, 1973). If a chunk representing a particular configuration of pieces is bound to a specific location on the board, more chunks would have to be acquired to achieve expertise than if chunks were free to match to the same configuration wherever they occurred on the board (Gobet \& Simon, 1996a; Saariluoma, 1984, 1994).

In answer to the first question, there is evidence that the closer two pieces are to each other on a board (i.e., their proximity), the more likely they are to be found in the same chunk (Chase \& Simon, 1973a; Gobet \& Simon, 1998). Gobet and Simon (1998) measured the temporal latency between piece replacements, assuming chunk boundaries corresponding to a 2-sec or longer latency between replacements. Their findings suggested that piece proximity was a determinant of chunk boundaries, with adjacent pieces more likely to be encoded together in a chunk. In answer to the second question, other research has indicated that the precise location of pieces is also encoded in chess chunks (Gobet \& Simon, 1996a; Saariluoma, 1984, 1994). These studies found lower recall performance for game positions with locationally transposed sections of pieces. These findings have influenced the structure of the CHREST model (de Groot \& Gobet, 1996; Gobet \& Simon, 2000). Although CHREST uses various types of information to constrain chunk acquisition, precise piece locations are always encoded, and one of the primary determinants by which pieces are grouped together into a chunk is spatial proximity.

However, it is possible that the stronger determinant of which pieces are put together in a chunk is the attack and defense relation between pieces: If a piece $\mathrm{A}$ is defending a piece $\mathrm{B}, \mathrm{A}$ and $\mathrm{B}$ are more likely to be in a chunk regardless of their proximity. This is consistent with an explanation given by Goldin (1979), who showed that more highly skilled players were much better than less skilled players at recognizing (as opposed to recalling) random chess positions. Saariluoma (1984) replicated this finding for shorter presentation times. Goldin (1979) suggested that this effect of skill was likely to be due to the use, by more highly skilled players, of memory structures for particular semantic features of the random positions. The effect of semantic features would be more evident in a recognition test than in a recall test (where an effect of expertise is much smaller), because less information may be required to make a simple discrimination than to reconstruct a position. Attack and defense relations are, of course, fundamentally important types of semantic features, since they are two of the foundational constraints in the game of chess.

Evidence supporting the importance of attack and defense relations to expert players' processing was reported by Saariluoma $(1984,1985)$. He found that more highly skilled players were quicker than less skilled players to respond correctly in the detection of direct attack and defense relations between pieces. Saariluoma's (1984, 1985) findings suggest that the basic ability to process these features with more efficiency is a fundamental and learned skill in chess-playing development. Saariluoma and colleagues (Saariluoma, 1990; Saariluoma \& Kalakoski, 1998) have also found evidence from memory performance that is consistent with the view that pieces are likely to be chunked in memory because of their functional significance. Skilled players had better recall for groups of pieces directly involved in a forcing combination of moves than for those pieces that had no particular function within the position. Saariluoma $(1990,1991)$ has argued that groups of pieces are extracted by skilled players and processed as important foregrounds, whereas all other pieces are considered a less important background. This is consistent with the view that pieces would be chunked in memory structures if the relations between those pieces were functionally important (see also Holding, 1985; Holding \& Reynolds, 1982; Saariluoma, 1995; Saariluoma \& Hohlfeld, 1994).

The plausibility that attack/defense relations have a greater role in determining chunk content is supported by Holding and Reynolds (1982). In their experiment, skilled players attempted both a recall task and an evaluation task with semirandom chess positions. Importantly, the results showed a dissociation between the ability to recall and the ability to evaluate positions. More highly skilled players produced significantly better evaluations of potential moves than did less skilled players, but there was no difference between the two skill groups' recall scores. Schultetus and Charness (1999) extended this finding, showing an effect of skill on recall of random positions only after participants had evaluated the presented positions. It is possible that when skilled chess players evaluate a position, as opposed to trying to remember it, they naturally elucidate the relations between pieces and, in so doing, activate relationally encoded information. 
In this article, we report three experiments designed to test the hypothesis that, during the evaluation of a board position, positions are encoded more in terms of the attack and defense relations between pieces than in terms of locations and proximity. In all three experiments, participants were asked to evaluate board positions prior to a memory test. In Experiment 1, the participants were presented with a series of artificial chess positions and were then given a recognition test and asked to discriminate between positions that had been presented and positions that had been either distorted by changing the attack/ defense relations between the pieces or shifted by moving all the pieces one square to the side. In Experiment 2, after the participants had evaluated positions taken from reported games, they were given a primed recognition test for pieces that were either proximal or related by attack/defense to the prime. Experiment 3 repeated Experiment 2 , but with the addition of a condition that manipulated the time given to the participants to evaluate a position. Following Gobet and Simon (2000), who reported an effect of presentation time on recall performance, it was hypothesized that the effects of attack/ defense relations on recognition would be apparent only after longer presentation times.

\section{EXPERIMENT 1}

Chess-playing participants were instructed to evaluate a series of positions, after which they were given a recognition test. Each test item either was presented as it had been in the evaluation phase or was altered, using a shift or distort transformation. In shift positions, all the pieces were moved one square horizontally from their original locations. In distort positions, a single piece was moved one square horizontally from its original location, and all the other pieces remained in their original places.

The experiment used longer presentation times than had many previous studies (e.g., Chase \& Simon, 1973a, 1973b; Gobet \& Simon, 1998). The 30-sec presentation time was used in order to allow the participants to adequately perform an evaluation task. We assumed that the task of evaluation requires a certain amount of processing time, consistent with the fact that, in actual chess play, individual positions can be studied for substantial periods of time, with an average of at least 2 min per position allowed by most chess tournament rules (cf. the claim by Gobet and Simon, 1996c, that reducing time available during play does not greatly reduce playing performance).

The experiment was designed to test the hypothesis that skilled chess participants would demonstrate a better ability to reject test items that contained different relations (i.e., ones that had been distorted) than to reject those that contained different locations (i.e., ones that had been shifted).

\section{Method}

Participants. Twenty-four participants, with varying degrees of chess-playing ability and experience, took part. Twelve were experienced club/tournament players, with a mean chess Elo (1978) grading of $1,906(S D=112)$. The other 12 participants were occasional players without regular chess-playing experience or gradings. The low-skill group consisted largely of undergraduate students; there were fewer students in the high-skill group.

Materials. Thirty simple chess positions were created, with all the positions constructed to be legal according to the laws of chess, such as checking and piece promotion. The positions contained a mean number of 7.5 pieces (the range varied between 5 and 9 pieces) and represented examples of the endgame stage of chess games, as opposed to opening or midgame positions.

In addition to the 30 original positions, two sets of altered positions were created by making two uniform transformations to original positions. The shift and distort transformations moved all the pieces or a single piece, respectively, one square horizontally in location. The direction of piece movement for the transformations was counterbalanced, with half of the movements to the square on the left and half to the right of the original square. Two transformations were generated from each original board position: one by shifting the pieces in the position and the other by distorting the position. Ten of the positions from the original set were used unaltered in the recognition task.

Positions were presented to participants by computer screen, using Superlab software, and appeared as $5 \times 5$ in. images. The timing of presentations was computer automated, and a keypress was used to move to the next position. The participants used paper to record evaluations of positions. For recognition responses, keypresses indicated yes and no choices.

Procedure and Design. Participants were given a sheet of instructions and were allowed to study a starting position depicting all of the pieces in the form they would appear during the experiment (e.g., so that a Queen could be distinguished from a Bishop). The participants were instructed that they would be presented with a series of positions, for $30 \mathrm{sec}$ each, during which time they should decide which color was winning or if there was no advantage. After $30 \mathrm{sec}$, the position disappeared from the screen and the participant was required to write down either the color they evaluated as winning or neutral. A prompt message followed each position on the screen, which stated that the next position would appear following a keypress. This process was completed for all 30 positions from the original set, with evaluations recorded for each position. The sequence of positions presented was randomly ordered by the computer for each participant.

For the recognition task, the participants were informed that they would be presented with a further 30 positions and were asked to indicate whether the position was one they had previously seen in the evaluation phase (old) or not (new). Positions were presented for a maximum of $90 \mathrm{sec}$, but the participants were asked to indicate their choice by a keypress as soon as they were satisfied with their answers. As before, following each position, a prompt indicated that a further keypress would present the next position.

During the recognition test, each participant was presented with equal numbers (10) of each type of test position. Thus, each participant was shown 10 shift positions, 10 distort positions, and 10 unchanged original positions from the stimulus set. The ordering of presentation was also randomized for this task.

The main dependent variable analyzed was the number of positions accepted as previously seen for the shift, distort, and original conditions during the recognition task. A secondary dependent variable analyzed was the response time of the participants during the recognition test.

The use of position and transformation type in the recognition task was balanced across participants by ensuring that half of the participants received a distorted version for a position $x$, whereas half saw position $x$ shifted. These two halves of the participants were yoked pairs matched for playing strength.

\section{Results}

A $2 \times 3$ mixed design analysis of variance (ANOVA) was carried out on the dependent measures of yes re- 
sponses made and the (correct) response times of the participants. Planned comparisons were made to examine the effects shown by the different skill groups.

Figure 1 shows the yes responses made by the participants in the three conditions. The main effect of transformation type was significant $\left[F(2,22)=8.10, M S_{\mathrm{e}}=\right.$ $4.20, p<.01]$. The interaction between transformation type and skill group was also significant $[F(2,22)=8.61$, $\left.M S_{\mathrm{e}}=4.20, p<.01\right]$. The main effect of skill group was not significant $(F<1)$.

A planned comparison $t$ test showed a significant difference between the number of yes responses made by the high-skill participants in the shift and the distort conditions $[t(44)=3.77, p<.01]$. The high-skill group made significantly fewer yes responses in the distort condition $(M=18.33 \%)$ than in the shift condition $(M=50.0 \%)$. A planned comparison $t$ test was significant for high-skill yes responses in the distort and original conditions $[t(44)=$ $5.65, p<.01]$, with significantly more yes responses in the original condition $(M=65.8 \%)$. There was no significant difference between the number of yes responses made by high-skill participants in the shift and the original conditions $[t(44)=1.88]$. No significant differences were found for the number of yes responses made by low-skill participants in the different recognition conditions (all $t \mathrm{~s}<1$ ).

Figure 2 shows the correct response times for all the participants in the three transformation conditions. The main effect of transformation type on (correct) response times was significant $\left[F(2,22)=5.86, M S_{\mathrm{e}}=15.40, p<\right.$ $.01]$. The main effect of skill level and the interaction between skill level and transformation condition were not significant $\left(F_{\mathrm{S}}<1\right)$.

A planned comparison $t$ test between response times in the shift and the distort conditions was significant $[t(44)=$ $2.27, p<.05]$. Correct response times were significantly quicker for the distort condition $(M=12.91 \mathrm{sec})$ than for the shift condition $(M=15.49 \mathrm{sec})$. A planned compari- son $t$ test between responses in the shift and original conditions was significant $[t(44)=3.35, p<.01]$, with shorter correct response times in the original condition $(M=$ $11.69 \mathrm{sec})$. A planned comparison $t$ test between response times in the distort and the original conditions did not show a significant difference $[t(44)=1.08]$. Analysis of the response times of the high-and the lowskill groups taken separately did not show any significant differences between the recognition conditions (all $t \mathrm{~s}<1)$.

\section{Discussion}

In summary, fewer yes responses were made for distort positions, in which the attack/defense relations between the pieces were new but locations were largely unchanged, than for shift positions, in which the attack/ defense relations were maintained but locations were entirely different.

The more highly skilled participants displayed over $80 \%$ accuracy in rejecting positions with different relations between the pieces, despite the small change between the originals studied and the distorted positions. It was also found that the skilled participants were faster to respond correctly to the distorted positions than to the shifted positions. The skilled participants were both quicker and more accurate in discriminating new relations between pieces than new locations of pieces.

In addition, we observed that the skilled participants took, on average, more than $10 \mathrm{sec}$ to respond to each test item. This is consistent with the fact that the experimental instructions emphasized accuracy over speed.

An interaction was observed between skill level and recognition condition. However, this interaction was due to a floor effect in the low-skill group performance and is not, therefore, meaningful. It is possible that low-skill players would display a different pattern of results with respect to skilled participants if given adequate opportunity. Regardless of the performance of the low-skill par-

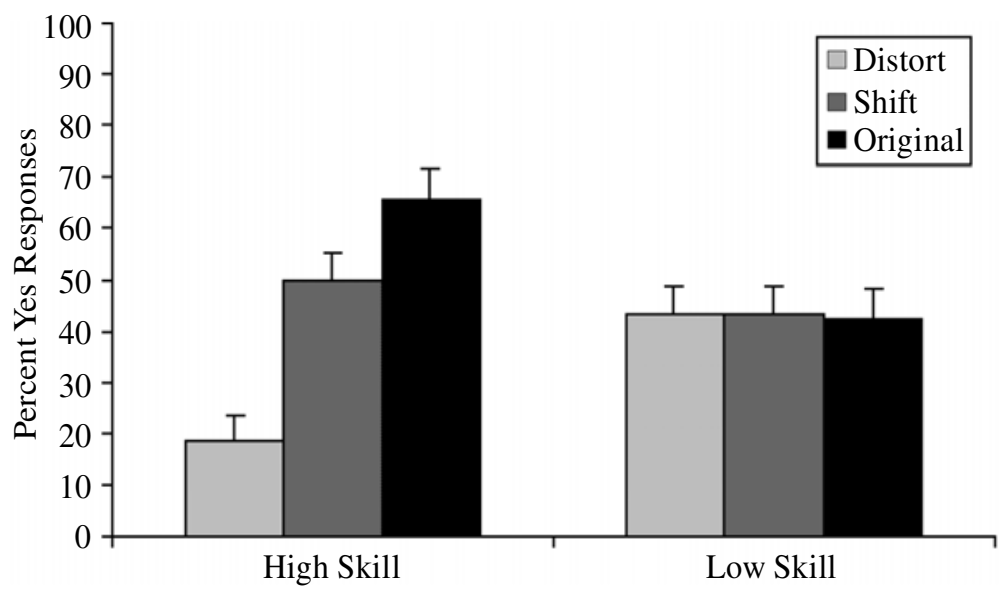

Figure 1. Experiment 1: percentage of yes responses as a function of skill group and recognition condition. The error bars are standard errors. 


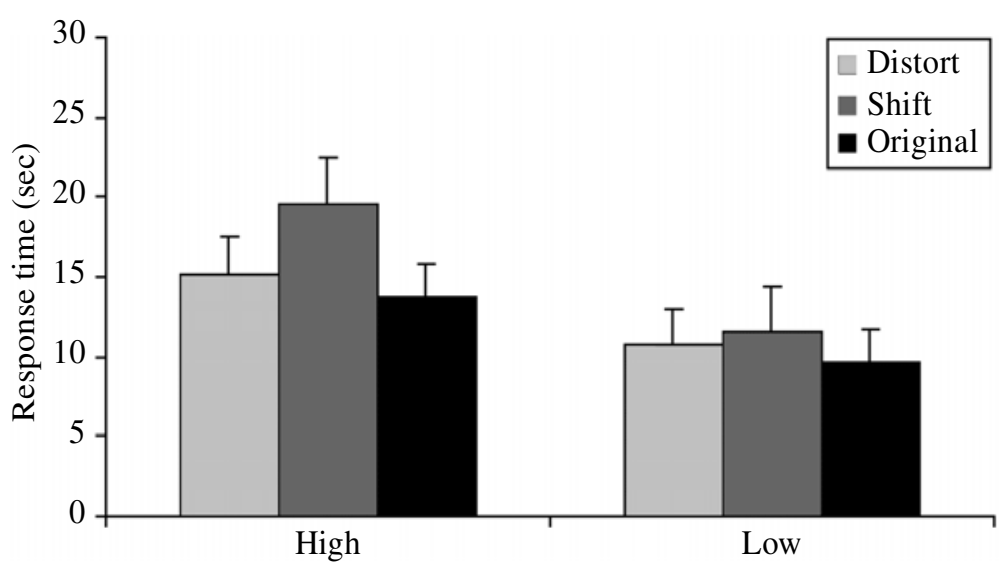

Figure 2. Experiment 1: response times (correct only) as a function of skill group and recognition condition. The error bars are standard errors.

ticipants, the performance of high-skill participants remains of interest. The performance of high-skill participants is consistent with the view that, during position evaluation, expert memory structures are encoded more in terms of the attack/defense relations between pieces than in terms of location. However, Experiment 1 does not provide evidence about the role of proximity relative to attack/defense relations. This issue was addressed in Experiment 2.

\section{EXPERIMENT 2}

Experiment 1 supported the view that skilled chess players encoded the attack/defense relations between pieces more accurately than they encoded their exact locations on the board. To extend the scope of this finding, Experiment 2 was designed to test for the role of attack/ defense relations between pieces, relative to the role of the proximity of pieces. In addition, stimuli were midgame positions taken from published master-level games.

It is important to establish the role of relational encoding relative to proximity, given previous evidence indicating that the proximity of pieces is a primary determinant of chunk boundaries (Chase \& Simon, 1973b; de Groot \& Gobet, 1996; Gobet \& Simon, 1998). Gobet and Simon (1998), following Chase and Simon's (1973b) design, asked participants to recall pieces from a briefly presented position. They measured the latencies between placements and assumed that latencies greater than $2 \mathrm{sec}$ corresponded to chunk boundaries. They found that the more constraints (same piece, same color, proximity, attack, and defense) that existed between two successively replaced pieces, the shorter the latency between replacements. Using this methodology, they also found that proximity was a stronger indicator of chunking between pieces than was the presence of attack and defense relations.

Chase and Simon's (1973b) study and subsequent replications already described provide strong evidence for the role of proximity in chunk coding. However, comparing Chase and Simon's (1973b) and Goldin's (1979) studies of memory for random positions indicates substantial differences between number of positions correctly remembered using recognition and recall tests (for random positions, an effect of skill is, for the most part, evident only in recognition). It is possible that the recall paradigm is more likely to give evidence for proximity simply because of the strategy used to perform the task. For example, when recalling positions, it may be better to go along rows or columns systematically to help ensure completeness.

In Experiment 2, participants were tested with a priming methodology. Following McNamara, Halpin, and Hardy (1992), we assumed that evidence of priming between items provides some indication of organization within memory. Generally, if Item A primes Item B, it can be assumed that A and B are closely associated in memory. We argue that the pattern of associations between chess pieces is important evidence to consider in determining the nature of skilled memory for chess. We assume that a chunking theory in which chunk content is determined more by the proximity of pieces predicts that, for skilled players, pieces that are closer together will be more likely to prime each other than will pieces that are far from one another. In contrast, if chunk content is determined more by the attack/defense relations between pieces, related pieces will prime each other regardless of the distance between them.

The participants were initially presented with a fullgame position and completed an evaluation task. Priming between pieces was assessed by measuring response times of the participants asked to recognize a target piece following the presentation of a prime piece from the previously seen position. The prime pieces either were directly related by attack/defense or had no direct relations (unrelated), and were spatially near or far from the target piece in the original position. 


\section{Method}

Participants. Twenty-four participants of varying chess-playing experience and ability took part in the experiment. Eight were regular club/tournament players with chess ratings, 8 were occasional players without regular playing experience, and 8 were not chess players. The mean grading for the regular-playing participants was $1,845(S D=120)$ on the Elo (1978) scale. These three groups were classified as high-skill, low-skill, and no-skill groups for the purposes of the study.

Materials. Twenty chess positions were taken from several sources of chess games, each position being one reached during actual games played by master-level players (mean number of pieces = 21.5). The positions were reconstructed on a Macintosh computer for presentation on the computer screen.

Graphics were created showing the board with just one of the pieces from each of the positions present, these single pieces being either prime or target pieces for the recognition tasks. For each position, one target piece was selected and shown in a graphic. Four different prime pieces were selected for each position, displaying four possible combinations of spatial proximity and relation to the target piece. The four priming piece combinations were related/near, related/far, unrelated/near, and unrelated/far in relation to the target piece. The near primes were pieces on squares adjacent to the target piece. The far primes were pieces a minimum of two squares distant $(M=4.2)$ from the target piece. Each relation was either a direct attack or a direct defense. Twenty graphics were also created showing a single incorrect piece in place of the target pieces, for use as foil trials.

The experiment was run using computer-timed presentation of graphics and recording of response times. Responses from the participants were recorded by keypresses indicating yes/no decisions. Evaluations of positions were recorded on paper.

Design. The participants were presented with all of the 20 fullboard positions for $30 \mathrm{sec}$ per position and were asked to perform an evaluation. Following each $30-\mathrm{sec}$ exposure to a full position, the chess-playing participants recorded an evaluation on paper of which color they thought held an advantage or whether the position was neutral (nonplayers recorded a tick). After writing their evaluations of the position, the participants were given a recognition test. A single piece from the position was presented on the board for $2 \mathrm{sec}$ as a priming piece. This piece was then removed from the screen, and a second piece was presented on the board. The participants were instructed not to respond to the presentation of the first piece and to indicate with a keypress whether they considered the second piece to be shown correctly. The participants were informed that the first piece presented was always a piece shown correctly from the position.
Each participant was given the recognition test with equal numbers of the four priming conditions, with 50\% correct and 50\% foil trials occurring. The order of trials was randomized by computer. Each participant was tested for correct-piece trials on a position only once. The use of different priming conditions for each specific position was balanced, with yoked participants of equal skill level receiving each of the four conditions per position.

\section{Results}

A $3 \times 2 \times 2$ mixed design ANOVA was carried out on the dependent variable of (correct) response times of participants to the target piece presentation. The independent variables were skill group, relatedness (related/ unrelated), and proximity of the prime (near/far). Planned comparisons were made on the effects of priming conditions for the different skill groups.

Figure 3 shows the participants' (correct) response times for all four combinations of priming condition. The main effects of skill group $[F(2,20)=1.20]$, relatedness $[F(1,20)=1.28]$, and proximity $(F<1)$ were not significant. There was a significant interaction between skill group and relatedness $\left[F(2,20)=5.71, M S_{\mathrm{e}}=3,019.80\right.$, $p<.05]$. The interaction between skill group and proximity was not significant $(F<1)$. The interaction between relatedness and proximity $(F<1)$ and the three-way interaction of skill group, relatedness, and proximity $[F(2,20)=1.24]$ were not significant.

A planned comparison $t$ test showed a significant effect of relatedness for the response times of the highskill group $[t(20)=2.44, p<.025]$. High-skill participants responded more quickly in the related trials than in the unrelated trials. There was no significant difference between response times of the other two skill groups for these two conditions (both $t \mathrm{~s}<1$ ).

A secondary analysis was performed on the number of errors made by the participants. The mean error rates on the piece recognition tests were $14.4 \%, 29.4 \%$, and $35.0 \%$ for the high-, low-, and no-skill groups, respectively. There was a main effect of skill group $[F(2,20)=$ $\left.8.95, M S_{\mathrm{e}}=0.97, p<.01\right]$. There was no main effect of

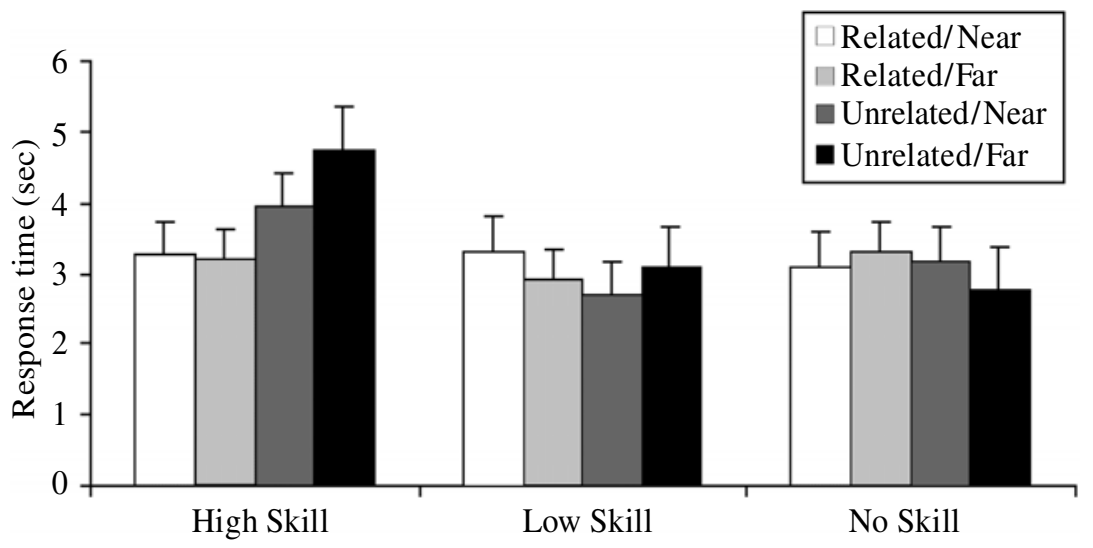

Figure 3. Experiment 2: response times (correct only) as a function of skill group and priming condition. The error bars are standard errors. 
relatedness or proximity $(F<1)$. There was, however, a significant interaction between relatedness and proximity for error rates $[F(1,20)=6.70, p<.05]$, with most errors occurring in the unrelated/far condition.

\section{Discussion}

On a recognition test, skilled chess players showed significantly faster responses to target pieces when they followed pieces that had been related by attack or defense in a studied position than when they followed pieces that had not been related. However, they did not show shorter response times when decisions were preceded with spatially proximal pieces than when they were preceded by spatially distant pieces. The low-skill players and no-skill players showed no evidence of differential priming between pieces.

The results were consistent with the view that the presence of a direct attack or defense relation between pieces in a position is more significant to the organization of skilled memory than is the proximity of pieces. The view that pieces with closer spatial proximity are more likely to be chunked in memory was not supported by the results.

Furthermore, the priming effect of direct relations was consistent with the findings of Saariluoma $(1984,1985)$ for the effect of expertise on simple relational processing. Saariluoma found that more highly skilled players were able to process the presence (or absence) of relations between two pieces more rapidly than were less skilled players, which implicated the adaptation of low-level processing of direct relations. The findings of Experiment 2 support the view that such relational processing becomes incorporated within the memory structures of skilled players and provides the criteria for association between particular pieces in memory.

One issue concerns the fact that the skilled players were not faster overall than the nonskilled players and that the skilled players were slower than were the unskilled players to process pieces not related by attack or defense. However, this is likely to be due to the fact that the nonskilled players guessed some responses in the absence of sufficient memory to support accurate decisions. This view is supported by the fact that the highskill players were, on average, twice as accurate as both the low-skill and the no-skill players.

Another issue concerns the fact that average response times were of the order of $4 \mathrm{sec}$ - that is, they cannot be described as immediate reaction times. We suspect that the explanation for this fact is that the participants were doing some strategic processing that would enable them to make a recognition decision. The results indicate that this processing made use of the prime but that additional processing was required.

\section{EXPERIMENT 3}

The previous two experiments reported in this article used a $30-\mathrm{sec}$ presentation of positions prior to memory testing. This presentation time is significantly longer than that used in many previous studies of chess memory (Chase \& Simon, 1973b; de Groot, 1965; Gobet \& Simon, 1996a, 1996b, 1998), which often have used briefer presentation times, of less than $10 \mathrm{sec}$, prior to immediate recall. The fact that there is an effect of skill on how much is remembered even at short presentation times has been used as evidence for memory structures that are immediately matched to the positions presented.

Together with the observation that differently skilled players do not exhibit reliably different search processes during evaluation (de Groot, 1965, 1966), the fact that high-skill players can recall a position after a brief presentation time has been used to support a model of game playing based on a recognition-association process between chunks and move solutions (Chase \& Simon, 1973a; Gobet, 1998; Gobet \& Jansen, 1994; Gobet \& Simon, 1996a, 1996d). The use of brief presentation times in previous studies has therefore been an important factor in the subsequent theory of both expert chess memory and chess game playing.

The possibility that presentation time influences the performance of skilled chess players is also suggested by more recent findings. Lories (1987) and Gobet and Simon (2000) both have found that by extending the presentation time of chess positions to $60 \mathrm{sec}$, expert chess players performed significantly better than less skilled players even in recall of random positions. In addition, a strong effect of skill has been found for recognition of random positions when participants were given more processing time (Goldin, 1979). These findings suggest that when time was available for positions to be processed to a greater depth, the memory structures of more highly skilled players were better suited to encoding random positions than were those of less skilled players. This interpretation is consistent with the study of Schultetus and Charness (1999), who found that the evaluative processing of random positions led to better memory for those positions. These findings are consistent with the view that evaluative processing of chess positions facilitates the representation of the positions in a skilled player's memory.

The previous literature on the effects of presentation time and the possibility that it facilitates deeper evaluation (i.e., more relational processing) led us to the prediction that longer presentation times would be more likely to enable highly skilled players to process attack and defense relations. Experiment 3 was therefore designed to test the effect of presentation time on recognition. The design and task of Experiment 2 were used, but with the addition of a presentation time condition ( 9 vs. $30 \mathrm{sec}$ ). For the shorter presentation time, a 9-sec period was used because it represented a substantial reduction from $30 \mathrm{sec}$ and because it was close to the upper limit of times used in previous studies (e.g., de Groot, 1965; Holding \& Reynolds, 1982). We hypothesized that the effect of attack/defense relations on memory, suggested by the results of Experiments 1 and 2, would not be found with a shorter presentation time. 


\section{Method}

Participants. Twenty-four participants, all able to play chess, took part in the experiment. Twelve participants were regular club/tournament players with at least 3 years playing experience and a mean grading of 1,832 $(S D=144)$ on the Elo scale. Twelve participants were occasional players without regular playing experience or off icial ratings. For this experiment, the former were classified as the high-skill group, and the latter were classified as the low-skill group.

Materials. Twenty chess positions, taken from actual games played by master-level players, were reproduced as graphics for presentation on a computer screen (mean number of pieces $=20.0$ ). From these positions, the prime and target graphics were created as described for Experiment 2, with related/near, related/far, unrelated/ near, and unrelated/far priming conditions. Twenty graphics were also created showing a single incorrect piece in place of the target pieces for use as foil trials.

The experiment was run using computer-timed presentation of graphics and recording of response times. Responses from the participants were recorded by keypresses indicating yes/no decisions. Evaluations of positions were recorded on paper.

Procedure. The procedure of Experiment 3 was identical to that described for Experiment 2, except for the presentation time of positions during the evaluation phase. Half of the participants (equally divided between skill groups) were presented with the positions during the evaluation task for $9 \mathrm{sec}$, and half were given $30 \mathrm{sec}$ for the evaluation phase. For the high-skill group, the participants were divided into yoked pairs with similar ratings (Elo, 1978), so that the different presentation time conditions were balanced for skill level. The low-skill group was yoked approximately according to the experience levels reported by the participants.

\section{Results}

A $2 \times 2 \times 2 \times 2$ mixed design ANOVA and two planned comparison $t$ tests were carried out on the dependent measure of correct response times to target pieces. The independent variables were skill group, presentation time, relatedness (related/unrelated), and proximity (near/ far) of the prime. Planned comparisons were made on the effects of priming condition and presentation times for the different skill groups.

Figure 4 shows the response times of participants for all the conditions. The main effect of presentation time was significant $\left[F(1,20)=12.39, M S_{\mathrm{e}}=16,169.35, p<\right.$ $.01]$. The response times were significantly shorter in the short presentation time condition than in the long presentation time condition. The main effects of skill group $(F<1)$, proximity $(F<1)$, and relatedness $[F(1,20)=$ 2.54] were not significant.

There was a significant interaction between skill group and relatedness $\left[F(1,20)=12.124, M S_{\mathrm{e}}=3,504.12\right.$, $p<.01]$. A planned comparison $t$ test showed a significant effect of relatedness for the response times of the high-skill participants $[t(20)=2.54, p<.02]$. High-skill participants were significantly quicker with related primes than with unrelated primes. No other interactions between skill, presentation time, relatedness, and proximity were significant (all $F_{\mathrm{s}}<1$ ).

Planned comparison $t$ test analyses were performed to assess whether the effect of relatedness was present for high-skill participants in the two different presentation times. High-skill participants' responses were quicker for related than for unrelated primes with the short presentation times $[t(20)=1.81, p<.05$, significant at the onetailed level]. Related condition responses were also quicker than unrelated ones for high-skill participants in the long presentation time condition $[t(20)=2.07, p<$ $.05]$.

The secondary measure of errors was also analyzed. The effect of skill group was significant for the number of recognition errors $\left[F(1,20)=20.964, M S_{\mathrm{e}}=0.91, p<\right.$ $.001]$. The high-skill group (17.5\%) made significantly fewer errors than did the low-skill group (35\%). All other main effects were not significant (all $F_{\mathrm{S}}<1$ ). A

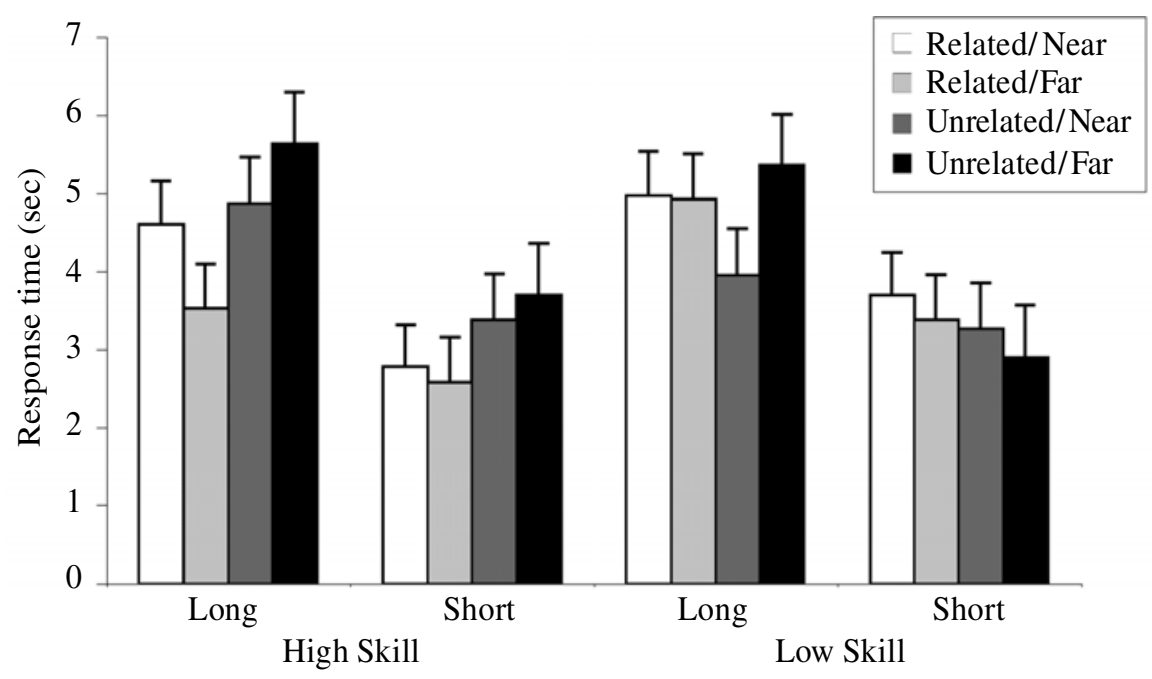

Figure 4. Experiment 3: response times (correct only) as a function of skill group, presentation time, and priming condition. The error bars are standard errors. 
four-way interaction between skill, presentation time, relatedness, and proximity was also significant for errors $\left[F(1,20)=6.097, M S_{\mathrm{e}}=0.49, p<.05\right]$.

\section{Discussion}

The main finding of Experiment 3 was that the memory priming effect for directly related pieces after a 30 -sec presentation time was also found when participants were given only a $9-\mathrm{sec}$ presentation time. The trials with related primes produced mean responses that were faster than those in the trials with unrelated primes, regardless of presentation time condition. The hypothesis that longer presentation times would lead to more relational priming was, therefore, not supported. The findings for the short presentation time condition support the view that memory for relations was activated rapidly when the positions were processed. In this respect, there appears to be no difference (for times between 9 and $30 \mathrm{sec}$ ) between the time needed to encode attack and defense relations and the time needed to encode proximity and location (Chase \& Simon, 1973a, 1973b; de Groot, 1965, 1966; Gobet, 1998; Gobet \& Simon, 1996a, 1996d).

We hypothesized that longer presentation times may be required to enable participants to evaluate a position and, in so doing, to encode attack and defense relations. However, the results of Experiment 3 suggest that attack and defense relations were encoded even for shorter presentation times. This result is in accordance with the findings of Saariluoma $(1984,1985)$ that experts are able to recognize the most basic relational features, such as check detection, more rapidly than are less skilled players. It seems that there is a continuum of evaluative processing, from which signs of relational encoding will be seen even at shorter presentation times.

It is possible that a greater reduction in presentation time, below the $9 \mathrm{sec}$ used here, may show a different pattern of results. At still shorter presentation times, participants may be unable to evaluate a position, and their test performance could then be more dependent on memory for location and proximity.

\section{GENERAL DISCUSSION}

In Experiment 1, the participants were presented with a series of chess positions and were asked to evaluate them. They were then given a recognition test and were asked to discriminate between positions that had been presented and positions that had been either distorted by changing the attack/defense relations between the pieces or shifted by moving all the pieces one square to the side. The results indicated that the skilled participants found it easier to avoid false recognition of positions that had been distorted than of positions that had been shifted. In Experiment 2, the participants were presented with chess positions taken from master-level games and were asked to evaluate them. After each position, they were given a priming test. The results showed that a piece that was re- lated to the target by attack or defense was more likely to prime memory than was a piece that was simply proximal to it. Experiment 3 repeated Experiment 2, but with the addition of a condition that manipulated the time given to participants to evaluate a position. The same pattern of results as that found in Experiment 2 was evident with the reduced presentation time.

These results provide an indication of how skilled players organize the information from chess positions in LTM structures that are activated when the players engage in the evaluation of a position. The results reported are consistent with the view that for more highly skilled participants, the structure of LTM chunks is determined more by the attack/defense relations between pieces than by the location or proximity of pieces.

Why did the present study expose evidence for memory structures that were encoded more in terms of attack/ defense relations, when previous studies have suggested that chunk boundaries were determined more by location and proximity (Chase \& Simon, 1973a, 1973b; de Groot, 1965; Gobet \& Simon, 1996a, 1996d, 1998; Saariluoma, 1994)? One answer to this question is that the participants were asked to perform different tasks in these experiments. Whereas, in the experiments reported in this article, the participants were asked to evaluate board positions, in many previous studies participants were asked to remember them (Chase \& Simon, 1973a, 1973b; de Groot, 1965; Gobet \& Simon, 1996a, 1996d, 1998; Saariluoma, 1994). It is likely that, as we have already argued, the process of position evaluation allows skilled players to take advantage of relational chunk content that is not made accessible by experimental designs that do not call on participants to evaluate a position (Chase \& Simon, 1973a, 1973b; de Groot, 1965; Gobet \& Simon, 1996a, 1996d, 1998; Saariluoma, 1994).

Another potential reason for the difference between our findings and those of previous experiments is that we used a recognition paradigm, whereas many previous studies have used a recall paradigm. Saariluoma (1984) compared the uses of recall and recognition in the study of chess expertise and emphasized that there were important differences in the results obtained from the two types of test. As we have said, whereas a large effect of skill has been shown for accuracy of recognition of random chess positions (Goldin, 1979; Saariluoma, 1984), the effect of skill for recall of random positions is negligible in comparison (Gobet \& Simon, 1996b). It is likely that the tasks of recall and recognition call for the use of different kinds of information. For example, it is worth noting that, for recall, players are required to reproduce the details of physical locations, whereas, for recognition, it is sufficient to consider only that evidence that leads to a yes/no discrimination. Perhaps, for recognition, the most diagnostic evidence is that which is derived from attack/defense relations. What is clear is that more investigation is required into the relation between the memory test used in an experiment and the relevance 
of the results obtained to the tasks in which players engage during game play. At present, we see no reason to consider recall tests to be superior to recognition tests, or vice versa.

The results of our experiments are consistent with our earlier interpretation of the results of Schultetus and Charness (1999), who found an effect of skill in memory for a random position following the performance of an evaluation task. We argue that the evaluative processing of positions allows activation of the attack and defense content of chunks, which, in turn, supports a more detailed encoding of random positions. In addition, our results may explain the findings of Lories (1987) and Gobet and Simon (2000) for recall of random positions when more time was given, if the assumption is adopted that the players performed some degree of evaluation. More highly skilled players would benefit from this evaluation in the same way as they did in the Schultetus and Charness experiment.

One possible limitation of our experiments is that they tested only the performance of skilled chess players on midgame and endgame type positions. In contrast to more relationally encoded memory structures, locationspecific encodings (Chase \& Simon, 1973a, 1973b; Gobet, 1998; Gobet \& Simon, 1996a, 1996d) may be more important at earlier stages of a game progression, owing to the greater degree of stereotypicality in openings and early midgame positions. This proposal is consistent with the reduced recall performance found when stereotypicality is artificially reduced (Goldin, 1978). The use of the attack and defense content of chunks may be important in novel positions and more developed games, which demand a degree of generalization (in terms of precise piece locations) from previously encountered situations.

Another consideration is that proximity and location may be more important to more highly skilled players than it was to those who participated in the present study. Several previous studies have investigated the performance of master-level participants (Chase \& Simon, 1973a, 1973b; de Groot, 1965; Gobet \& Simon, 1996a, 1996d, 1998, 2000), as opposed to the lower level of playing expertise possessed by our high-skill participants. Further research would be necessary to discover whether the development of master-level expertise would lead to a difference in the pattern of recognition results reported in this article.

We have argued that attack/defense relations are more important determinants of chunk content than proximity and location, but we also acknowledge previous findings in favor of proximity and location. It is possible that individual chunks may contain information about all three kinds of constraint (attack/defense, proximity, and location). Another possibility is that one type of chunk contains information about proximity and location and another about attack and defense and that these chunks are organized into a hierarchy. Although the evidence presented in this article is not sufficient to distinguish these positions, the latter view is consistent with the hierarchical approach to expert memory, such as that proposed by Vicente and Wang (1998). In their constraint attunement hypothesis, they proposed the view that experts' memory structures will be constrained to represent different levels of goal-relevant information from the domain. This, in turn, suggests that expert memory is likely to consist of several different types of representation.

The importance of attack/defense relations in determining chunk content is recognized by Gobet and Simon (2000). They reported a model that incorporates an encoding mechanism based on a model of a chess player's eye movements. The model makes use of several mechanisms for determining where to fixate next. Included in these mechanisms is one in which "the fixation of a square following a relation of attack or defence" (p. 670) is used. However, they also state that "each fixation delineates a visual field (all squares within $+/-$ two squares from the square fixated), and the pieces belonging to this visual field are sorted through the discrimination net" (p. 670). This second aspect of the model implies that 25 pieces may be encoded in chunks purely because of their proximity to the fixated piece. As a consequence, and in conflict with our findings, Gobet and Simon's (2000) model does not appear to predict the relative effects on recognition memory of attack/defense relations over proximity and location, observed in our experiments.

We introduced the topic of this article with two questions: (1) Which chess pieces are combined into chunks with which others and (2) whether chunks are encoded with information specific to a particular board location (the square on the board). In summary, we have argued that, for experts, the influence of attack and defense relations on memory for a position is intimately bound to the process of skilled evaluation. Our experiments support the view that when skilled chess players engage in the evaluation of a chess position, they make use of chunks encoded more in terms of attack/defense relations between pieces than in terms of proximity or location. These findings challenge theories of expertise for chess that assume a primary role for proximity and location in determining which pieces are grouped together in memory.

\section{REFERENCES}

Chase, W. G., \& Simon, H. A. (1973a). The mind's eye in chess. In W. G. Chase (Ed.), Visual information processing (pp. 215-281). New York: Academic Press.

Chase, W. G., \& Simon, H. A. (1973b). Perception in chess. Cognitive Psychology, 4, 55-81.

DE Groot, A. D. (1965). Thought and choice in chess. The Hague: Mouton.

DE GRoot, A. D. (1966). Perception and memory versus thought: Some old ideas and recent findings. In B. Kleinmuntz (Ed.), Problem solving: Research, method and theory (pp. 19-50). New York: Wiley. DE Groot, A. D., \& Gobet, F. (1996). Perception and memory in chess: Heuristics of the professional eye. Assen, The Netherlands: Van Gorcum.

ELo, A. (1978). The rating of chess players, past and present. New York: Arco. 
Gobet, F. (1998). Expert memory: A comparison of four theories. Cognition, 66, 115-152.

Gobet, F., \& JANSEN, P. (1994). Towards a chess program based on a model of human memory. In H. J. Herik, I. S. Herschberg, \& J. W. Uiterwijk (Eds.), Advances in computer chess 7 (pp. 35-60). Maastricht: University of Limburg Press.

Gobet, F., \& Simon, H. A. (1996a). Recall of random and distorted chess positions: Implications for the theory of expertise. Memory \& Cognition, 24, 493-503.

GobET, F., \& Simon, H. A. (1996b). Recall of rapidly presented random chess positions is a function of skill. Psychonomic Bulletin \& Review, 3, 159-163.

Gobet, F., \& Simon, H. A. (1996c). The roles of recognition processes and look-ahead search in time-constrained expert problem-solving: Evidence from grandmaster level chess. Psychological Science, 7, 52-55.

Gobet, F., \& SimON,H. A. (1996d). Templates in chess memory: A mechanism for recalling several boards. Cognitive Psychology, 31, 1-40.

Gobet, F., \& Simon, H. A. (1998). Expert chess memory: Revisiting the chunking hypothesis. Memory, 6, 225-255.

Gobet, F., \& Simon, H. A. (2000). Five seconds or sixty? Presentation time in expert memory. Cognitive Science, 24, 651-682.

Goldin, S. E. (1978). Memory for the ordinary: Typicality effects in chess memory. Journal of Experimental Psychology: Human Learning \& Memory, 104, 605-611.

GoLDIN, S. E. (1979). Recognition memory for chess positions: Some preliminary research. American Journal of Psychology, 92, 19-31.

HoLding, D. H. (1985). The psychology of chess skill. Hillsdale, NJ: Erlbaum.

Holding, D. H., \& Reynolds, R. I. (1982). Recall or evaluation of chess positions as determinants of chess skill. Memory \& Cognition, 10, 237-242.

LORIES, G. (1987). Recall of random and non-random chess positions in strong and weak chess players. Psychologica Belgica, 27, 153-159.

McNamara, T. P., Halpin, J. A., \& Hardy, J. K. (1992). Spatial and temporal contributions to the structure of spatial memory. Journal of
Experimental Psychology: Learning, Memory, \& Cognition, 18, 555564.

Miller, G. A. (1956). The magical number seven, plus or minus two: Some limits on our capacity for processing information. Psychological Review, 63, 81-97.

SAARILUOMA, P. (1984). Coding problem spaces in chess: A psychological study (Commentationes Scientarum Socialium, No. 23). Helsinki: Societas Scientarum Socialium.

SAARILUOMA,P. (1985). Chess players' intake of task-relevant cues. Memory \& Cognition, 13, 385-391.

SAARILUOMA, P. (1990). Apperception and restructuring in chess players' problem solving. In K. J. Gilhooly, M. T. G. Keane, R. H. Logie, \& G. Erdos (Eds.), Lines of thinking (Vol. 2, pp. 41-57). New York: Wiley.

SAariluoma, P. (1991). Aspects of skilled imagery in blindfold chess. Acta Psychologica, 77, 65-89.

SAARILUOMA, P. (1994). Location coding in chess. Quarterly Journal of Experimental Psychology, 47A, 631-650.

SAARILUOMA, P. (1995). Chess players' thinking: A cognitive psychological approach. London: Routledge.

SaARiluoma, P., \& Hohlfeld, M. (1994). Apperception in chess players' long-range planning. European Journal of Cognitive Psychology, 6, 1-22.

SAARILUOMA, P., \& KaLAKosKI, V. (1998). Apperception and imagery in blindfold chess. Memory, 6, 67-90.

Schultetus, R. S., \& Charness, N. (1999). Recall or evaluation of chess positions revisited: The relationship between memory and evaluation in chess skill. American Journal of Psychology, 112, 555-569.

Simon, H. A., \& Gilmartin, K. J. (1973). A simulation of memory for chess positions. Cognitive Psychology, 5, 29-46.

VICENTE, K. J., \& WANG, J. H. (1998). An ecological theory of expertise effects in memory recall. Psychological Review, 105, 33-37.

(Manuscript received December 18, 1998; revision accepted for publication April 3, 2002.) 